\title{
Boundary Domination of Line and Middle Graph of Wheel Graph Families
}

\author{
Mohammed Alatif \\ Department of Mathematics \\ P.E.S.College of Engineering, \\ Mandya-571 401. India
}

\author{
Puttaswamy Rangaiah \\ Department of Mathematics \\ P.E.S.College of Engineering, \\ Mandya-571 401. India
}

\author{
Nayaka S.R. \\ Department of Mathematics \\ P.E.S.College of Engineering, \\ Mandya-571 401.India
}

\begin{abstract}
Let $G=(V, E)$ be a connected graph. A subset $\mathrm{S}$ of $V(G)$ is called a boundary dominating set if every vertex of $V-S$ is boundary dominated by some vertex of $S$. The minimum taken over all boundary dominating sets of a graph $G$ is called the boundary domination number of $G$ and is denoted by $\gamma_{b}(G)$. We define the boundary domatic number in graphs. Exact values of of Wheel Graph Families are obtained and some other interesting results are established.
\end{abstract}

\section{Keywords}

Boundary dominating set, Boundary domination number, Boundary domatic number

\section{INTRODUCTION}

For graph-theoretical terminology and notations not defined here we follow Buckley [2] and Haynes et al.[4]. Let $\mathrm{G}$ be a nontrivial connected graph. The distance between two vertices $u$ and $v$ is the length of a shortest path joining them. The eccentricity $e(u)$ of a vertex $u$ is the distance to a vertex farthest from $u$. A vertex $v$ is called an eccentric vertex of $u$ if $e(u)=d(u, v)$. A vertex $v$ is an eccentric vertex of $G$ if $v$ is an eccentric vertex of some vertex of $G$. Consequently if $v$ is an eccentric vertex of $u$ and $w$ is a neighbor of $v$, then $d(u, w) \leq d(u, v)$. A vertex $v$ may have this property, however, without being an eccentric vertex of $u$. Let $G$ be a simple graph $G=(V, E)$ with vertex set $V(G)=\left\{v_{1}, v_{2}, \ldots, v_{n}\right\}$. For $i \neq j$, a vertex $v_{i}$ is a boundary vertex of $v_{j}$ if $d\left(v_{j}, v_{t}\right) \leq d\left(v_{j}, v_{i}\right)$ for all $v_{t} \in N\left(v_{i}\right)$ [3].

A vertex $v$ is called a boundary neighbor of $u$ if $v$ is a nearest boundary of $u$. If $u \in V$, then the boundary neighbourhood of $u$ denoted by $N_{b}(u)$ is defined as $N_{b}(u)=\{v \in V: d(u, w) \leq$ $d(u, v)$ for all $w \in N(u)\}$. The cardinality of $N_{b}(u)$ is denoted by $\operatorname{deg}_{b}(u)$ in $G$. The maximum and minimum boundary degree of a vertex in $G$ are denoted respectively by $\Delta_{b}(G)$ and $\delta_{b}(G)$. That is $\Delta_{b}(G)=\max _{u \in V}\left|N_{b}(u)\right|, \delta_{b}(G)=\min _{u \in V}\left|N_{b}(u)\right|$.

A vertex $u$ boundary dominate a vertex $v$ if $v$ is a boundary neighbor of $u$. KM. Kathiresan, G. Marimuthu and M. Sivanandha Saraswathy [5] introduced the concept of Boundary domination in graphs. Puttaswamy and Mohammed Alatif [6] introduced the concept of Boundary edge domination in graphs. All graphs considered in this paper are finite and contains no loops and no multiple edges. For a real number $x ;\lfloor x\rfloor$ denotes the greatest integer less than or equal to $x$ and $\lceil x\rceil$ denotes the smallest integer greater than or equal to $x$.

Line graph $L(G)$ of a graph $G$ is defined with the vertex set $E(G)$, in which two vertices are adjacent if and only if the corresponding edges are adjacent in $G$.

Middle graph $M(G)$ of a graph $G$ is defined with the vertex set $V(G) \cup E(G)$, in which two elements are adjacent if and only if either both are adjacent edges in $G$ or one of the elements is a vertex and the other one is an edge incident to the vertex in $G$. We need the following theorems.

THEOREM 1. [6] If $G$ is a connected graph of size $m \geq 3$, then $\left\lceil\frac{m}{\Delta_{b}^{\prime}+1}\right\rceil \leq \gamma_{b}^{\prime}(G) \leq m-\Delta_{b}^{\prime}(G)$.

Theorem 2. [6] For any $(n, m)$-graph $G, \gamma^{\prime}(G)+\gamma_{b}^{\prime}(G) \leq$ $m+1$.

THEOREM 3. For any gear graph $G_{n}$ with $n>3, \gamma\left(G_{n}\right)=$ $\left\lceil\frac{n}{2}\right\rceil+1$.

THEOREM 4. [1] For any helm graph $H_{n}$ with $n>3$, $\gamma\left(H_{n}\right)=n$.

THEOREM 5. For any connected graph $G, d_{b}(G) \leq\left\lfloor\frac{n}{\gamma_{b}(G)}\right\rfloor$.

\section{RESULTS}

\subsection{Boundary Domination In Graphs}

DEFINITION 6. A subset $S$ of $V(G)$ is called a boundary dominating set if every vertex of $V-S$ is boundary dominated by some vertex of $S$. The minimum taken over all boundary dominating sets of a graph $G$ is called the boundary domination number of $G$ and is denoted by $\gamma_{b}(G), \gamma_{b}^{\prime}(G)$ for the line graph of $G$ and $\gamma_{b}(M(G)$ ) for the middle graph of $G$.

2.1.1 Wheel Graph. The wheel graph $W_{n}$ on $n+1$ vertices is defined as $W_{n}=C_{n}+K_{1}$ where $C_{n}$ is $n-$ cycle. Let $V\left(W_{n}\right)=$ $\left\{v_{i}: 1 \leq i \leq n\right\} \cup\{v\}$ and $E\left(W_{n}\right)=\left\{e_{i}=v_{i} v_{i+1}, 1 \leq i \leq n\right.$, subscripts modulo $n\} \cup\left\{e_{i}^{\prime}=v v_{i}, 1 \leq i \leq n\right\}$, where $v$ is an external vertex adjacent to every other vertex.

THEOREM 7. For any wheel graph $W_{n}, \gamma_{b}\left(W_{n}\right)=1$

PROOF. Let $W_{n}$ be a wheel graph of order $n+1$. Since $d\left(v, v_{1}\right)=d\left(v, v_{2}\right)=\ldots=d\left(v, v_{n}\right)=1$, then $N_{b}(v)=$ $\left\{v_{1}, v_{2}, \ldots, v_{n}\right\}, \delta_{b}=\Delta_{b}=n$ so that $S=\{v\}$ and $|S|=1$. Hence 


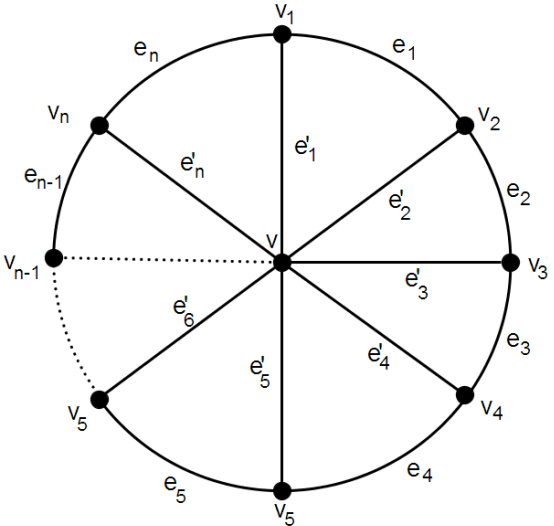

Fig. 1. Wheel graph $W_{n}$

$\gamma_{b}\left(W_{n}\right) \leq 1$. Further since $\gamma_{b}\left(W_{n}\right) \geq\left\lceil\frac{n+1}{\Delta_{b}+1}\right\rceil=\left\lceil\frac{n+1}{n+1}\right\rceil=1$. Thus $\gamma_{b}\left(W_{n}\right)=1$.

THEOREM 8. For a wheel graph $W_{n}, n \geq 3, \gamma_{b}^{\prime}\left(W_{n}\right)=3$.

Proof. Let $L\left(W_{n}\right)$ be the line graph of $W_{n}$ of order $2 n$ Since $d\left(e_{i}, e_{i+1}\right) \leq d\left(e_{i}, e_{i+2}\right), d\left(e_{i}, e_{i-1}\right) \leq d\left(e_{i}, e_{i-2}\right)$ for all $e_{i+1}, e_{i-1} \in N\left(e_{i}\right)$ and $e_{i+2}, e_{i-2} \in N_{b}\left(e_{i}\right)$, also $d\left(e_{i}, e_{i}^{\prime}\right) \leq$ $d\left(e_{i}, e_{i-1}^{\prime}\right), d\left(e_{i}, e_{i+1}^{\prime}\right) \leq d\left(e_{i}, e_{i+2}^{\prime}\right)$ for all $e_{i}^{\prime}, e_{i+1}^{\prime} \in N\left(e_{i}\right)$ and $e_{i-1}^{\prime}, e_{i+2}^{\prime} \in N_{b}^{\prime}\left(e_{i}\right)$ so that $\delta_{b}^{\prime}=n-2, \Delta_{b}^{\prime}=n$, and for $1 \leq$ $i \leq n$, the cycle $C_{3}=\left\{e_{i}, e_{i}^{\prime}, e_{i+1}^{\prime}\right\}$ or $\left\{e_{i-1}, e_{i}, e_{i}^{\prime}\right\}$ is a boundary edge dominating set of $W_{n}$. Hence $|S|=\gamma_{b}^{\prime}\left(W_{n}\right)=3$.

THEOREM 9. For a wheel graph $W_{n}, n \geq 3, \gamma_{b}\left(M\left(W_{n}\right)\right)=$

Proof. The proof is similar to the proof of Theorem 2.3.

2.1.2 Gear Graph. The gear graph is a wheel graph with vertices added between pair of vertices of the outer cycle. The gear graph $G_{n}$ has $2 n+1$ vertices and $3 n$ edges. Let $V\left(G_{n}\right)=\left\{v_{i}: 1 \leq i \leq\right.$ $n\} \cup\left\{u_{i}: 1 \leq i \leq n\right\} \cup\{v\}$ and $E\left(G_{n}\right)=\left\{e_{i}=v_{i} u_{i}, 1 \leq i \leq\right.$ $n\} \cup\left\{e_{i}^{\prime}=v_{i} v, 1 \leq i \leq n\right\} \cup\left\{e_{i}^{\prime \prime}=u_{i} v_{i+1}, 1 \leq i \leq n\right.$, subscripts modulo $n\}$, where $v$ is an external vertex adjacent to every other vertex $v_{i}$ for $1 \leq i \leq n$.

THEOREM 10. For any gear graph $G_{n}, \gamma_{b}\left(G_{n}\right)=2$.

Proof. Let $X, Y$ be a bipartition of $G_{n}$, with $X=$ $\left\{v_{1}, v_{2}, \ldots, v_{n}\right\}$ and $Y=\left\{u_{1}, u_{2}, \ldots, u_{n}\right\} \cup\{v\}$. Let $v_{i} \in X$. Then $d\left(v_{i}, v_{j}\right)=2$ for all $v_{j} \in X-\left\{v_{i}\right\} ; i \neq j$ and every vertex $v_{j}$ in $X$ is a boundary neighbour of $v_{i}$ except $v_{i}$. Similarly $d\left(v, u_{i}\right)=2$ , then every vertex of $Y-\{v\}$ is a boundary neighbour of $u_{i}$ except $v$ and $\delta_{b}^{\prime}=\Delta_{b}^{\prime}=n$ therefore $S=\left\{v, v_{i}\right\}$, is a boundary dominating set of $G_{n}$ for all $i$ so that $|S|=2$. Hence $\gamma_{b}\left(G_{n}\right) \leq 2$. Further since $\Delta_{b}=n, \gamma_{b}\left(G_{n}\right) \geq\left\lceil\frac{2 n+1}{\Delta_{b}+1}\right\rceil=\left\lceil\frac{2 n+1}{n+1}\right\rceil$, then $\gamma_{b}\left(G_{n}\right) \geq 2$. Hence $\gamma_{b}\left(G_{n}\right)=2$.

THEOREM 11. For a gear graph $G_{n}, \gamma_{b}^{\prime}\left(G_{n}\right)=3$.

Proof. Let $L\left(G_{n}\right)$ be the line graph of $G n$ of order $3 n$. Since $d\left(e_{i}, e_{i}^{\prime}\right) \leq d\left(e_{i}, e_{i+1}^{\prime}\right)$,

$d\left(e_{i}, e_{i}^{\prime \prime}\right) \leq d\left(e_{i}, e_{i+1}\right)$ for all $e_{i}^{\prime}, e_{i}^{\prime \prime} \in N\left(e_{i}\right)$ and $e_{i+1}^{\prime}, e_{i+1} \in$

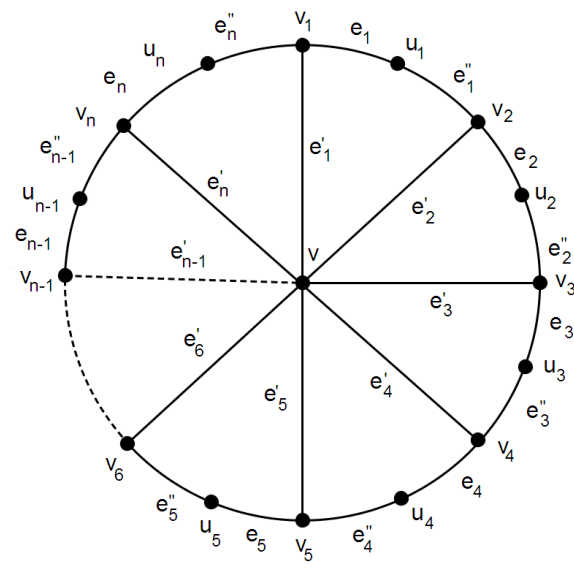

Fig. 2. $G_{n}$

$N_{b}\left(e_{i}\right)$, similarly $e_{i}, e_{i}^{\prime \prime} \in N^{\prime}\left(e_{i}^{\prime}\right)$ and $e_{i+1}, e_{i+1}^{\prime \prime} \in N_{b}^{\prime}\left(e_{i}^{\prime}\right)$ so that $\delta_{b}^{\prime}=n+1, \Delta_{b}^{\prime}=2 n-2$, and for $1 \leq i \leq n$, the cycle $C_{3}=\left\{e_{i}, e_{i}^{\prime}, e_{i}^{\prime \prime}\right\}=S$ is a boundary edge dominating set of $G_{n}$ and $|S|=3$. Hence $\gamma_{b}^{\prime}\left(G_{n}\right) \leq 3$. Further since the collection $\left\{e_{i}, e_{i}^{\prime}, e_{i}^{\prime \prime}: 1 \leq i \leq n\right\}$ contains n-cycles of order 3 then $|S| \geq\left\lceil\frac{3 n}{n}\right\rceil=3$ so that $\gamma_{b}^{\prime}\left(G_{n}\right) \geq 3$. Thus $\gamma_{b}^{\prime}\left(G_{n}\right)=3$

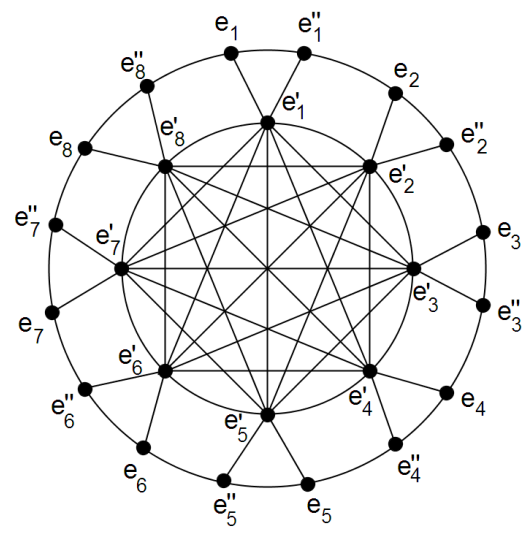

Fig. 3. $L\left(G_{8}\right)$

THEOREM 12. For a gear graph $G_{n}, \gamma_{b}\left(M\left(G_{n}\right)\right)=\left\lceil\frac{n}{2}\right\rceil+1$.

Proof. Let $M\left(G_{n}\right)$ be the middle graph of $G_{n}$ of order $5 n+1$. Since $d\left(e_{i}, e_{i}^{\prime}\right) \leq d\left(e_{i}, e_{i+1}^{\prime}\right), d\left(e_{i}, e_{i}^{\prime \prime}\right) \leq d\left(e_{i}, e_{i+1}\right)$, for all $e_{i}^{\prime}, e_{i}^{\prime \prime} \in N\left(e_{i}\right), e_{i+1}^{\prime \prime}$, and $e_{i+1}^{\prime}, e_{i+1} \in N_{b}\left(e_{i}\right)$. Similarly $e_{i}^{\prime}, e_{i}^{\prime \prime}, e_{i} \in N\left(v_{i}\right)$ and $e_{i+1}^{\prime}, e_{i+1}^{\prime \prime}, u_{i} \in N_{b}\left(v_{i}\right)$ so that $\delta_{b}=n+4, \Delta_{b}=3 n$ and for $1 \leq i \leq n$ the set $S=\left\{e_{i}^{\prime}\right.$ : $\left.i=2 k+1, k<\left\lceil\frac{n}{2}\right\rceil\right\} \cup\left\{v_{1}\right\}$ is is a boundary dominating set of $M\left(G_{n}\right)$ and $|S|=\left\lceil\frac{n}{2}\right\rceil+1$. Hence $\gamma_{b}\left(M\left(G_{n}\right)\right) \leq\left\lceil\frac{n}{2}\right\rceil+1$. Further any boundary dominating set of $M\left(G_{n}\right)$ must contains at least one of $e_{i}^{\prime}, v_{i}$ for all $i$ and hence $|S| \geq n \geq\left\lceil\frac{n}{2}\right\rceil+1$ so that $\gamma_{b}\left(M\left(G_{n}\right)\right) \geq\left\lceil\frac{n}{2}\right\rceil+1$. Thus $\gamma_{b}\left(M\left(G_{n}\right)\right)=\left\lceil\frac{n}{2}\right\rceil+1$. 
2.1.3 Helm Graph. The helm graph $H_{n}$ is the graph obtained from an $n$-wheel graph by adjoining a pendant edge at each node of the cycle. The helm graph $H_{n}$ has $2 n+1$ vertices and $3 n$ edges and $V\left(G_{n}\right)=\{v\} \cup\left\{v_{i}: 1 \leq i \leq n\right\} \cup\left\{u_{i}: 1 \leq i \leq n\right\}$ and $E\left(G_{n}\right)=\left\{e_{i}=v_{i} v_{i+1}, 1 \leq i \leq n-1\right\} \cup\left\{e_{i}^{\prime}=v_{i} v, 1 \leq i \leq\right.$ $n-1\} \cup\left\{e_{i}=v_{i} u_{i}, 1 \leq i \leq n-1\right.$. where $v$ is an external vertex adjacent to every other vertex $v_{i}$ for $1 \leq i \leq n$

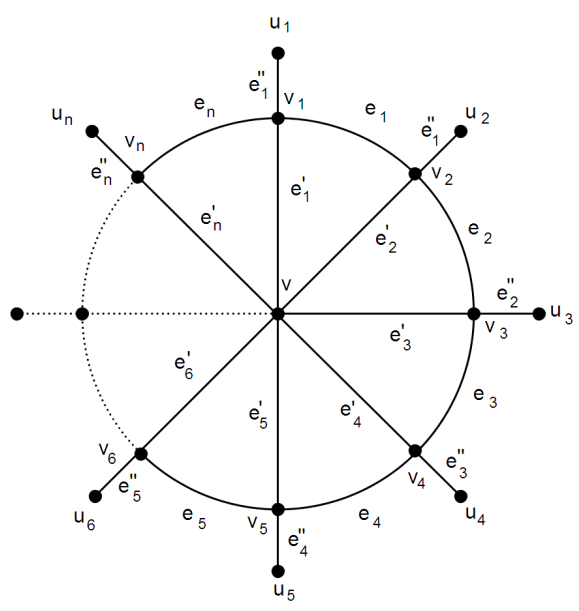

Fig. 4. $H_{n}$

THEOREM 13. For any helm graph $H_{n}, \gamma_{b}\left(H_{n}\right)=3$.

Proof. Let $(X, Y)$ be a bipartition of $H_{n}$, with $X=$ $\left\{v_{1}, v_{2}, \ldots, v_{n}\right\}$ and $Y=\left\{u_{1}, u_{2}, \ldots, u_{n}\right\} \cup\{v\}$. Let $u_{i} \in Y$. Since $d\left(v, u_{i}\right)=2$, then every vertex of $Y-\{v\}$ is a boundary neighbour of $u_{i}$ except $v$ and $\Delta_{b}=n$. Similarly since $d\left(v_{i}, v\right) \leq d\left(v_{i}, v_{i+2}\right)$ for all $v, v_{i+1} \in N\left(v_{i}\right)$, then every vertex $v_{j}$ in $X$ for $i+2 \leq j<n$ is a boundary neighbour of $v_{i}$ except $v_{i}$, also $v_{i+1}$ is a boundary neighbour of $v_{i-1}$ except $v_{i}, v_{i+1}$ and $\delta_{b}^{\prime}=2$, so that for all $i$ the set $S=\left\{v, v_{i}, v_{i+1}\right\}$ is a boundary dominating set of $H_{n}$ where $S=\left\{v, v_{1}, v_{2}\right\}$ or $\left\{v, v_{2}, v_{3}\right\}$ or $\ldots\left\{v, v_{n-1}, v_{n}\right\}$ and $|S|=3$. Hence $\gamma_{b}\left(H_{n}\right)=3$.

THEOREM 14. For any helm graph $H_{n}$,

$$
\gamma_{b}^{\prime}\left(H_{n}\right)=\left\{\begin{array}{c}
2 \text { if } n=3 \text { or } 4 \\
3 \quad \text { otherwise }
\end{array} .\right.
$$

Proof. The result is obvious if $n=3$ or 4 . Suppose $n \geq 5$, Since $d\left(e_{i}, e_{i}^{\prime}\right) \leq d\left(e_{i}, e_{i+2}^{\prime}\right), d\left(e_{i}, e_{i}^{\prime \prime}\right) \leq d\left(e_{i}, e_{i+2}\right)$ for all $e_{i}^{\prime}, e_{i}^{\prime \prime} \in N\left(e_{i}\right)$ and $e_{i+2}^{\prime}, e_{i+2} \in N_{b}\left(e_{i}\right)$, similarly $e_{i}, e_{i}^{\prime \prime} \in N^{\prime}\left(e_{i}^{\prime}\right)$ and $e_{i+1}, e_{i+1}^{\prime \prime} \in N_{b}^{\prime}\left(e_{i}^{\prime}\right)$ so that $\delta_{b}^{\prime}=n+2, \Delta_{b}^{\prime}=2 n-3$, and for $1 \leq i \leq n$, the set $S=\left\{e_{i}^{\prime}, e_{i}, e_{i+1}^{\prime}\right\}$ is a boundary edge dominating set of $H_{n}$ and $|S|=3$. Hence $\gamma_{b}^{\prime}\left(H_{n}\right) \leq 3$. Further since the collection $\left\{e_{i}^{\prime}, e_{i}, e_{i+1}^{\prime}: 1 \leq i \leq n\right\}$ contains n-cycles of order 3 then $|S| \geq\left\lceil\frac{3 n}{n}\right\rceil=3$ so that $\gamma_{b}^{\prime}\left(H_{n}\right) \geq 3$. Thus $\gamma_{b}^{\prime}\left(H_{n}\right)=3$

THEOREM 15. For a helm graph $H_{n}, \gamma_{b}\left(M\left(H_{n}\right)\right)=\left\lceil\frac{n}{2}\right\rceil+1$.

PROOF. The proof is similar to the proof of Theorem 2.7.

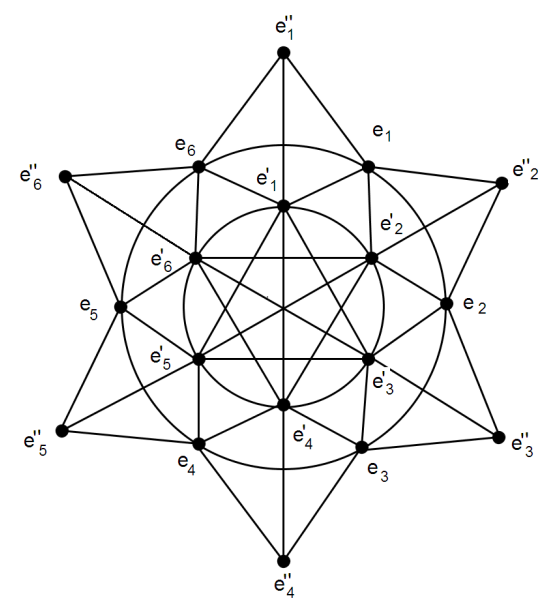

Fig. 5. $\quad L\left(H_{6}\right)$

\subsection{Boundary Domatic Number}

The maximum order of a partition of the vertex set $\mathrm{V}$ of a graph $\mathrm{G}$ into dominating sets is called the domatic number of $\mathrm{G}$ and is denoted by $d(G)$. For a survey of results on domatic number and their variants we refer to Zelinka [7]. In this section we present a few basic results on the boundary domatic number of a graph.

DEFINITION 16. Let $G=(V, E)$ be a connected graph. The maximum order of a partition of $V$ into boundary dominating sets of $G$ is called the boundary domatic number of $G$ and is denoted by $d_{b}(G)$.

THEOREM 17.,$d_{b}\left(W_{n}\right)=d_{b}\left(G_{n}\right)=d_{b}\left(H_{n}\right)=1$.

THEOREM 18. For a wheel graph $W_{n}, n \geq 3, d_{b}^{\prime}\left(W_{n}\right)=$ $\left\lceil\frac{2 n}{3}\right\rceil$.

Proof. By the definition of line graph, $V\left(L\left(W_{n}\right)\right)=$ $E\left(W_{n}\right)=\left\{e_{i}=v_{i} v_{i+1}, 1 \leq i \leq n\right.$, subscripts modulo $\left.n\right\}$ $\cup\left\{e_{i}^{\prime}=v v_{i}, 1 \leq i \leq n\right\}$. Let

$$
C=\left\{e_{i} e_{i}^{\prime} e_{i+1}^{\prime}: i=3(k-1), 1 \leq k \leq\left\lceil\frac{2 n}{3}\right\rceil\right\} .
$$

and

$$
C^{\prime}=\left\{e_{i} e_{i+1} e_{i+1}^{\prime}: i=3 k-2,1 \leq k \leq\left\lceil\frac{2 n}{3}\right\rceil\right\} .
$$

be a collection of 3-cycles of $L\left(W_{n}\right)$. Clearly the cycles of $C$ and $C^{\prime}$ are vertex disjoint and if $V(C)$ and $V\left(C^{\prime}\right)$ denotes the set of vertices belonging to the cycles of $C$ and $C^{\prime}$ respectively then $V(C) \cap V\left(C^{\prime}\right)=\phi$. Hence $d_{b}^{\prime}\left(W_{n}\right) \geq|C|+\left|C^{\prime}\right|=2\left\lceil\frac{n}{3}\right\rceil$. If $n \equiv 0$ or $1(\bmod 3)$, then $2\left\lceil\frac{n}{3}\right\rceil=\left\lceil\frac{2 n}{3}\right\rceil$ and $d_{b}^{\prime}\left(W_{n}\right) \geq\left\lceil\frac{2 n}{3}\right\rceil$.

If $n \equiv 2(\bmod 3)$, then $\left\lceil\frac{2 n}{3}\right\rceil=2\left\lceil\frac{n}{3}\right\rceil+1$. In this case $e_{n-2}^{\prime}, e_{n-1}^{\prime}, e_{n-2}, e_{n-1} \notin V(C) \cup V\left(C^{\prime}\right)$ and the set $\left\{e_{n-2}^{\prime}, e_{n-1}^{\prime}, e_{n-2}\right\}$ induces a 3 -cycle. Hence if $n \equiv 2(\bmod 3)$ $d_{b}^{\prime}\left(W_{n}\right) \geq 2\left\lceil\frac{n}{3}\right\rceil+1=\left\lceil\frac{2 n}{3}\right\rceil$. Therefore in both the cases $d_{b}^{\prime}\left(W_{n}\right) \geq\left\lceil\frac{2 n}{3}\right\rceil$. Also since $V\left(L\left(W_{n}\right)\right)=2 n$ and $\gamma_{b}^{\prime}\left(W_{n}\right)=3$, we have $\bar{d}_{b}^{\prime}\left(W_{n}\right) \leq \frac{2 n}{\gamma_{b}^{\prime}} \leq\left\lceil\frac{2 n}{3}\right\rceil$. Hence $d_{b}^{\prime}\left(W_{n}\right)=\left\lceil\frac{2 n}{3}\right\rceil$.

THEOREM 19. For a wheel graph $W_{n}$ and its middle graph $M\left(W_{n}\right)$, 


$$
d_{b}\left(M\left(W_{n}\right)\right)=\left\{\begin{array}{l}
2 \quad \text { if } n=3 \\
n \text { otherwise }
\end{array} .\right.
$$

Proof. The result is obvious if $n=3$, Suppose $n \geq 4$ by the definition of middle graph $V(M(G))=V(G) \cup E(G)$, and since $\left|V\left(M\left(W_{n}\right)\right)\right|=3 n+1, \gamma_{b}\left(M\left(W_{n}\right)\right)=3$, then $d_{b}\left(M\left(W_{n}\right)\right) \leq$ $\frac{3 n+1}{\gamma_{b}} \leq\left\lfloor\frac{3 n+1}{3}\right\rfloor \leq n$. Further let $C=\left\{P_{i}=v_{i} e_{i}^{\prime} e_{i+2}^{\prime}: 1 \leq\right.$ $i \leq n\}$ be the collection of paths of $M\left(W_{n}\right)$. Clearly the paths of $C$ are vertex disjoint and $|C|=n$, then $d_{b}\left(M\left(W_{n}\right)\right) \geq n$. Hence $d_{b}\left(M\left(W_{n}\right)\right)=n$

THEOREM 20. For a gear graph $G_{n}, d_{b}^{\prime}\left(G_{n}\right)=n$.

Proof. let $L\left(G_{n}\right)$ be a line graph of gear graph of order $3 n$, since $\gamma_{b}^{\prime}\left(G_{n}\right)=3$, it follows that $d_{b}^{\prime}\left(G_{n}\right) \leq\left\lfloor\frac{3 n}{\gamma_{c b}^{\prime}}\right\rfloor=\left\lfloor\frac{3 n}{3}\right\rfloor=n$.

To prove the reverse inequality, let $\Gamma=\left\{e_{i}, e_{i}^{\prime}, e_{i}^{\prime \prime}: 1 \leq i \leq n\right\}$ be a partition of the set of cycles of $L\left(G_{n}\right)$. It is clear that the cycles of $\Gamma$ are vertex disjoint and $|\Gamma|=n$ therefore $d_{b}^{\prime}\left(G_{n}\right) \geq n$. Hence $d_{b}^{\prime}\left(G_{n}\right)=n$.

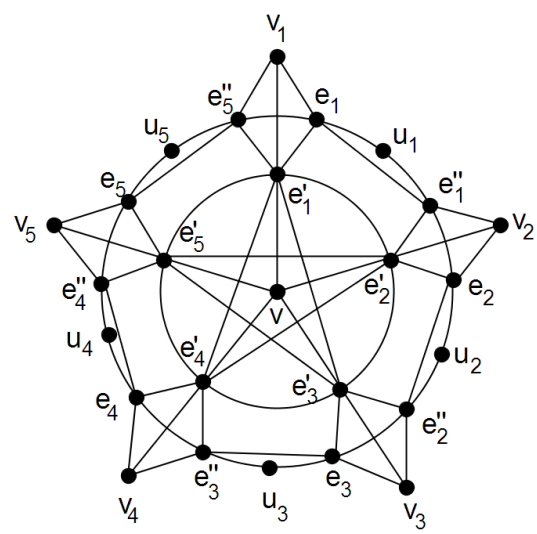

Fig. 6. $M\left(G_{5}\right)$

THEOREM 21. For a gear graph $G_{n}$,

$$
d_{b}^{\prime}\left(M\left(G_{n}\right)\right)=\left\{\begin{array}{cc}
n+1 & \text { if } n \leq 5 \\
n & \text { if } n=6 \text { or } 7 \\
4 & \text { if } n=8 \text { or } 9 \\
2 & \text { otherwise }
\end{array}\right.
$$

ProOF. The result is obvious if $n \leq 9$. In otherwise, by the definition of middle graph, $V\left(M\left(G_{n}\right)\right)=V\left(G_{n}\right) \cup$ $E\left(G_{n}\right),\left|V\left(M\left(G_{n}\right)\right)\right|=5 n+1$ in which the set $\left\{e_{i}^{\prime}: 1 \leq\right.$ $i \leq n\} \cup\{v\}$ induces a clique $K_{n+1}$ of order $n+1$ and for each $i,(1 \leq i \leq n)$, the set of vertices $\left\{e_{i}^{\prime \prime}, e_{i+1}^{\prime}, e_{i+1}, v_{i+1}:\right.$ subscript modulo $n\}$ induce a clique of order 4 . Also Since $\operatorname{deg}_{b}\left(u_{i}\right)=4$ and $\left|N_{b}\left(u_{i}\right): 1 \leq i \leq n\right|=4 n$, then $d_{b}^{\prime}\left(M\left(G_{n}\right) \leq\left\lceil\frac{5 n+1}{4 n}\right\rceil=2\right.$. To prove the reverse inequality, we consider the following cases.

Case $1 n$ is even

Let $S_{1}=\left\{v_{i}: i=2 k+1,0 \leq k<\left\lfloor\frac{n}{3}\right\rfloor\right\} \cup\left\{e_{n-3}, e_{n-2}^{\prime}, e_{n-2}^{\prime \prime}\right\}$ and $S_{2}=\left\{v_{i}: i=2 k, 1 \leq k \leq\left\lfloor\frac{n}{3}\right\rfloor\right\} \cup\left\{e_{n-2}, e_{n-1}^{\prime}, e_{n-1}^{\prime \prime}\right\}$. Clearly $\left\{S_{1}, S_{2}\right\}$ is a boundary domatic partition of $M\left(G_{n}\right)$ so that $d_{b}^{\prime}\left(M\left(G_{n}\right) \geq 2\right.$.

Case $2 n$ is odd

Let $S_{1}=\left\{v_{i}: i=2 k+1,0 \leq k \leq\left\lfloor\frac{n}{3}\right\rfloor\right\} \cup\left\{e_{n-3}, e_{n-2}^{\prime}, e_{n-2}^{\prime \prime}\right\}$ and $S_{2}=\left\{v_{i}: i=2 k, 1 \leq k \leq\left\lceil\frac{n}{3}\right\rceil\right\} \cup\left\{e_{n-2}, e_{n-1}^{\prime}, e_{n-1}^{\prime \prime}\right\}$. Clearly $\left\{S_{1}, S_{2}\right\}$ is a boundary domatic partition of $M\left(G_{n}\right)$ so that $d_{b}^{\prime}\left(M\left(G_{n}\right) \geq 2\right.$. Thus $d_{b}^{\prime}\left(M\left(G_{n}\right)=2\right.$

THEOREM 22. For a helm graph $H_{n}, d_{b}^{\prime}\left(H_{n}\right)=n$.

PROOF. The proof is similar to the proof of Theorem 2.15.

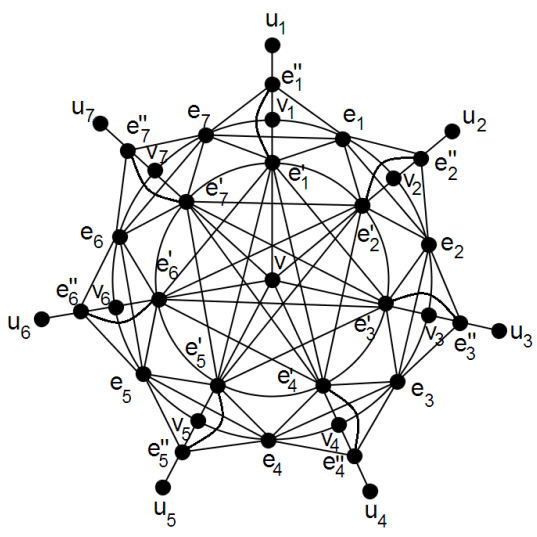

Fig. 7. $\quad M\left(H_{7}\right)$

THEOREM 23. For a helm graph $H_{n}$,

$$
d_{b}^{\prime}\left(M\left(H_{n}\right)\right)=\left\{\begin{array}{l}
5 \quad \text { if } n=5 \\
3 \text { if } n=7 \text { or } 9 \\
2 \quad \text { otherwise }
\end{array} .\right.
$$

Proof. The result is obvious if $n=5,7$ or 9 . In otherwise, by the definition of middle graph, $V\left(M\left(H_{n}\right)\right)=V\left(H_{n}\right) \cup$ $E\left(H_{n}\right),\left|V\left(M\left(H_{n}\right)\right)\right|=5 n+1$ in which for each $i,(1 \leq i \leq n)$, the set of vertices $\left\{e_{i}, e_{i+1}, e_{i+1}^{\prime}, e_{i+1}^{\prime \prime}, v_{i+1}\right.$ : subscript modulo $n\}$ induce a clique of order 5. Also $\left\{e_{i}^{\prime}: 1 \leq i \leq n\right\} \cup\{v\}$ induces a clique of order $n+1$ (say $K_{n+1}$ ). Since $\operatorname{deg}_{b}\left(u_{i}\right)=4$ and $\left|N_{b}\left(u_{i}\right): 1 \leq i \leq n\right|=3 n+1$, then $d_{b}^{\prime}\left(M\left(H_{n}\right) \leq\left\lceil\frac{5 n+1}{3 n+1}\right\rceil=2\right.$. To prove the reverse inequality, we consider the following cases.

\section{Case $1 n$ is even}

Let $S_{1}=\left\{e_{i}: i=2 k+1,0 \leq k \leq\left\lceil\frac{n}{2}\right\rceil+1\right\} \cup\left\{e_{n-1}^{\prime}, v_{n}\right\}$ and $S_{2}=\left\{e_{i}: i=2 k, 1 \leq k \leq n-2\right\} \cup\left\{v_{1}, e_{n}^{\prime}\right\}$. Clearly $\left\{S_{1}, S_{2}\right\}$ is a boundary domatic partition of $M\left(H_{n}\right)$ so that $d_{b}^{\prime}\left(M\left(H_{n}\right) \geq\right.$ 2 .

Case $2 n$ is odd

Let $S_{1}=\left\{e_{i}: i=2 k+1,0 \leq k \leq\left\lceil\frac{n}{2}\right\rceil+2\right\} \cup\left\{u_{n-2}, e_{n-1}^{\prime}, v_{n}\right\}$ and $S_{2}=\left\{e_{i}: i=2 k, 1 \leq k \leq n-3\right\} \cup\left\{u_{n-1}, e_{n}^{\prime}, v_{1}\right\}$. Clearly $\left\{S_{1}, S_{2}\right\}$ is a boundary domatic partition of $M\left(H_{n}\right)$ so that $d_{b}^{\prime}\left(M\left(H_{n}\right) \geq 2\right.$. Thus $d_{b}^{\prime}\left(M\left(H_{n}\right)=2\right.$ 


\section{CONCLUSION}

In this paper we computed the exact value of the boundary domination number and the boundary domatic number for the Wheel Graph Families, line graph of Wheel Graph Families and middle of Wheel Graph Families .

\section{REFERENCES}

[1] Ayhan A. khalil, Determination and Testing the Domination Numbers of Helm Graph, J. Edu. and Sci, Vol. (24), No. (2) 2011.

[2] F. Buckley and F. Harary, Distance in Graphs, AddisonWesley Reading, 1990.

[3] G. Chartrand, David Erwin, G. L. Johns and P. Zhang, On boundary vertices in graphs, J. Combin. Math. Combin.Comput., 48 (2004), 39-53.

[4] T.W. Haynes, S.T. Hedetniemi and P.J. Slater, Fundamentals of Domination in Graphs, Marcel Dekker, Inc., New York, (1997).

[5] KM. Kathiresan, G. Marimuthu and M. Sivanandha Saraswathy, Boundary domination in graphs, Kragujevac J. Math. 33 (2010) 63-70.

[6] Puttaswamy and Mohammed Alatif , Boundary edge domination in graphs, Bulletin Of the International Mathematical Virtual Instiute. 5(2015)197-204

[7] B. Zelinka, Edge domatic number of a graph, Czech. Math. J., 33(108)(1983), 107-110. 